\title{
Experiencias de partos en casa y en el hospital de mujeres en los años 60 y 70 en España
}

\section{Experiences of home births and hospital births of women in the 60s and 70s years in Spain Experiências de partos domiciliares e partos hospitalares de mulheres nos anos 60 e 70 na Espanha}

\author{
Celia Driéguez Castañoํㅜ Esther María López Martínez², Zaraida \\ Avilés Sáez ${ }^{3}$, María Belén Conesa Ferrer ${ }^{4}$ \\ ${ }^{1}$ Graduada en Enfermería en la Universidad de Murcia \\ ${ }^{2}$ Graduada en Enfermería en la Universidad de Murcia \\ ${ }^{3}$ Graduada en Enfermería en la Universidad de Murcia \\ ${ }^{4}$ Matrona y profesora asociada de la Universidad de Murcia
}

Cómo citar este artículo en edición digital: Driéguez Castaño, C., López Martínez, E. M. ․ Avilés Sáez, A. E Conesa Ferrer, M. ํㅡ. B. (2018). Experiencias de partos en casa y en el hospital de mujeres en los años 60 y 70 en España. Cultura de los Cuidados (Edición digital), 22(52). Recuperado de http://dx.doi.org/10.14198/cuid.2018.52.07

Correspondencia: C/ Rosario, n. $\stackrel{\circ}{5}$, dúplex 1. Santo Ángel, Murcia (España). C. P.: 30151

Correo electrónico: celia.drieguez@um.es

Recibido: 11/04/2018; Aceptado: 23/05/2018

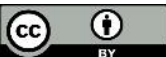

\section{ABSTRACT}

The main purpose of the study is to describe the experience of women who lived their births in hospitals and at home in Spain between 60's and 70's years. For women, childbirth is one of the most special moments of their lives, hence the importance of knowing the feelings experienced in this process, which will help us to improve this care on future occasions and know what women really value when giving birth. This study also reflected that home births, provided they are low risk and serviced by qualified personnel, are safe and we should be left to the woman who, with all the information, decide always where she want to give birth. This is a qualitative research based on Grounded Theory.
Keywords: Home birth, Hospital birth, Breastfeeding, Induction, Midwife.

\section{RESUMO}

O principal objetivo do estudo é descrever a experiência de mulheres que viveram suas entregas em hospitais e em casa em Espanha entre 60-70. Para as mulheres, o parto é um dos momentos mais especiais de suas vidas, daí a importância de se conhecer os sentimentos vivenciados neste processo, o que vai nos ajudar a melhorar essa assistência em ocasiões futuras e saber o que as mulheres realmente valor quando o parto. Este estudo também refletiu que partos domiciliares, desde que sejam de baixo risco e servido por pessoal qualificado, são seguros e quais devem ser deixadas para a 
mulher que, com todas as informações, decidir sempre onde você deseja entregar. Esta é uma pesquisa qualitativa, baseada na Teoria Fundamentada.

Palavras chave: Parto em casa, Parto hospitalar, Lactância materna, Indução, Matrona.

\section{RESUMEN}

El objetivo principal del estudio es describir la experiencia de las mujeres que vivieron sus partos en el entorno hospitalario y en casa, en España entre los años 60-70. Para las mujeres, el parto es uno de los momentos más especiales de sus vidas, de ahí la importancia de conocer los sentimientos experimentados en este proceso, lo que nos ayudará a mejorar estos cuidados en futuras ocasiones y saber qué es lo que las mujeres realmente valoran a la hora de dar a luz. En este estudio también queda reflejado que los partos en casa, siempre que sean de bajo riesgo y atendidos por personal cualificado, son seguros y que hay que dejar a la mujer que, con toda la información, decida siempre donde quiere dar a luz. Se trata de una investigación cualitativa basada en la Teoría Fundamentada.

Palabras clave: Parto en casa, Parto en el hospital, Lactancia materna, Inducción, Matrona.

\section{INTRODUCCIÓN}

Antiguamente el parto era considerado un proceso natural, el cual se llevaba a cabo en el hogar de la parturienta con ayuda de otras mujeres. Sin embargo, como era un medio inseguro por la escasez de medidas higiénicas, lo que daba lugar a una gran morbimortalidad de la madre y el neonato, durante el siglo XX, comenzó a institucionalizarse y medicalizarse el parto. La evolución de la humanidad, la ciencia y la tecnología, unido a los cambios de vida de las sociedades, si bien es cierto que han contribuido de manera importante a reducir los riesgos para la salud y elevar la calidad de vida, también ha hecho que procesos tan naturales como el parto se asuman como vivencias mucho más complejas y se elimine toda la naturalidad que lo caracteriza (Arnau, 2012). Actualmente el modelo de atención obstétrica está fuertemente institucionalizado y consta de un gran intervencionismo médico. Esto se debe a que los factores dolor y riesgo se consideran eventos patológicos que necesitan ser controlados para eliminar cualquier amenaza que pueda interferir en el embarazo y el parto. Esta situación ha tenido repercusiones negativas en la manera que la mujer experimenta la vivencia de su embarazo, parto y puerperio. De alguna manera, deja de ser la protagonista del proceso, viviendo estas experiencias como algo negativo (Arnau, 2012). Por lo tanto, surge la necesidad a nivel mundial de un cambio en la vivencia de la maternidad donde exista una mirada integral, teniendo en cuenta no sólo aspectos biológicos, sino también sus componentes psicológicos, familiares y sociales. Esto aumentará la calidez y humanidad del proceso. Esta propuesta de humanización parte de concebir el nacimiento como un proceso normal, que involucra a ambos miembros de la pareja y destaca la importancia de propiciar un ambiente relajante y familiar que favorezca la intimidad y la libre evolución del proceso (Arnau, 2012). Es decir, el parto, como experiencia única $\mathrm{y}$ probablemente la más significativa para la mujer, debería ser, en principio, un proceso fisiológico que únicamente necesita ser observado, en la mayoría de los casos. Se 
debe respetar su evolución, aplicando con sensatez y prudencia los recursos humanos y tecnológicos disponibles. De este modo se preserva el equilibrio entre la seguridad de la mujer y el feto y se respeta un proceso natural (De Miguel y Odriozola, 2010). Este tipo de parto, es una buena opción para más de un $80 \%$ de mujeres sin complicaciones médicas durante la gestación. En la actualidad, aunque esto está empezando a cambiar, el parto en casa se ha normalizado en países como Holanda y es una prestación pública más. Eso sí, con personal y medios especializados. También están proliferando en Suiza y Nueva Zelanda casas de nacimiento, centros cálidos dotados de personal y medios ideados sólo para partos.

En España, el parto en casa es una opción a la que opta una minoría y de forma privada. Pero en otras épocas de la historia no fue así. Las mujeres en España daban a luz en casa y fue a partir del siglo XIX cuando se produjo el cambio del entorno del parto del hogar familiar al hospital.

En los años 60 y 70 en España, la situación económica mejoró de forma notable, aunque desigual, el nivel de vida de la mayoría de la población, que formó una clase media hasta entonces casi inexistente. El nivel de libertad personal y política no aumentó del mismo modo. Empezaron las movilizaciones de oposición a la dictadura por parte de trabajadores y estudiantes. En cuanto a la sanidad, aumentaron las prestaciones sanitarias y se alargaba cada vez más la esperanza de vida, a la vez que disminuía la tasa de mortalidad.

Respecto a las matronas, durante las décadas centrales del siglo $X X$ acontecieron grandes cambios culturales que afectaron tanto a la población en general como a la profesión en particular. Nuevas leyes regularon los itinerarios y requisitos formativos para las matronas. Además, paulatinamente se produjo la institucionalización del parto de manera que los nacimientos pasaron de ser un acontecimiento propio del ámbito privado del hogar a integrarse como proceso hospitalario. Existen estudios sobre los profesionales que vivieron el cambio de la asistencia de los partos en casa al hospital (Rodríguez, 2014).

En la revisión bibliográfica realizada previa al planteamiento del estudio se pudo constatar que existen numerosos estudios centrados en aspectos cuantitativos del parto, pero no es así cuando lo que queremos es investigar sobre los sentimientos que experimentan las mujeres durante este proceso tan importante de su vida. Por eso el acercarnos a esta época de la historia de España desde la vivencia de las mujeres que experimentaron tanto el tener el parto en casa como en el hospital, puede ser muy interesante. Se planteó como objetivo principal del trabajo conocer la experiencia de las mujeres que habían vivido este cambio en la asistencia del parto (de la casa al hospital) y qué supuso para ellas.

\section{MATERIAL Y MÉTODOS Diseño del estudio}

Se trata de un diseño de investigación de carácter cualitativo basada en la Teoría Fundamentada debido a que nos permite entender mejor la naturaleza del comportamiento humano mediante la generación de teorías sobre fenómenos psicosociales (Arantzamendi, Gordo, LópezDicastillo y Vivar, 2010).

\section{Procedimientos para la recogida de datos}

Como estrategia principal para la recogida de datos se utilizó la entrevista semiestructurada en profundidad 
(Wordpress, 2008).

Con el objeto de delimitar previamente los objetos de interés, se diseñaron una serie de preguntas y se conformó un guion (Anexo I). Sin embargo, se les dio a las mujeres libertad para expresar todo lo que ellas considerasen importante. Las entrevistas se realizaron en los domicilios de las entrevistadas (Anexo II), seis de ellas en la zona de Balsicas, San Javier, Santiago de la Ribera y San Pedro del Pinatar y dos de ellas en el barrio de Vistabella de Murcia. Solo una de las entrevistadas se encontraba sola, las otras ocho estaban acompañadas por su marido, o por otro miembro de la unidad familiar, que también aportó datos en algún momento dado de la entrevista. El periodo de recogida de la información fue desde el 15 de enero hasta el 3 de febrero de 2016.

\section{Población y muestra}

Población: mujeres que han tenido experiencias de partos en casa y en el hospital con residencia en la región de Murcia. Muestra: El tipo de muestreo fue en bola de nieve.

Criterios de inclusión: Mujeres que hayan tenido al menos un parto en casa y un parto en el hospital, edades comprendidas entre los años 60 y 70 y residencia en la Región de Murcia.

Criterios de exclusión: Alteración cognitiva que dificulte recordar datos del pasado.

En primer lugar se explicaba a las entrevistadas el objeto y las características de la investigación y posteriormente se solicitaba su consentimiento informado por escrito (Anexo III). A partir de ese momento se llevaba a cabo la entrevista.

\section{Procedimientos para el análisis de datos}

En primer lugar se procedió a transcribir la información de las entrevistas, recogida a través de grabaciones de audio.

Para el análisis, se utilizaron los recursos del software Atlas.ti 7.5.10 y los principios del Análisis Temático de Contenido. En el pre-análisis se creó, en el software Atlas.ti, un archivo de almacenamiento [Unidad hermenéutica], en el que se introdujeron los datos de la investigación, obtenidos mediante entrevistas y observación [Documentos primarios]. Para cada entrevista [documento] se le asignó un número único. El material se examinó sobre la base de la selección/recorte de las unidades de significado, creación y asignación de códigos (77), que se agruparon en categorías (5) y éstas a su vez se agruparon en familias (2). Finalmente, para la interpretación, se llevaron a cabo asociaciones entre códigos, categorías y familias generando redes de visualización que fueron extraídas del software y presentadas en los resultados. Cabe señalar también que el proceso de análisis en todas sus fases se fundamentó en una tríada cíclica, a saber: los datos empíricos, la fundamentación y la percepción de los investigadores sobre el fenómeno estudiado. Este proceso en definitiva es un proceso de síntesis que permite ir de los aspectos más concretos a los más amplios.

\section{RESULTADOS}

En el nivel de codificación abierta emergieron 5 categorías referentes a distintos aspectos del parto: "Acompañamiento, Dolor, Lugar del parto, Profesional que asiste al parto, y Técnicas usadas en el parto". Las categorías se agruparon en dos familias: "Parto en casa y Parto en hospital". A continuación se exponen los resultados divididos según las dos familias descritas. 


\section{Parto en casa}

En el anexo IV se recogen las gráficas correspondientes a las categorías de la familia "Parto en casa".

\subsection{Acompañamiento (Gráfico 1)}

En cuanto a los sentimientos de la mujer, el que pudieran estar acompañadas por sus familiares en el parto en la casa es una de las principales razones de preferencia de este lugar ya que les hacía sentirse mejor:

CI: 1. Muy bueno, muy bueno, muy bueno. Excelente, excelente. Porque estaba con mi madre, en mi casa, el ambiente no tenía nada que ver. Me acuerdo yo que sí, que mucho mejor que en el hospital. Mucho mejor.

Con respecto a las personas que acompañaban a las mujeres en los partos en casa eran generalmente mujeres, o las madres, suegras, cuñadas, vecinas y en menor medida los maridos:

CI: 2. Mi madre estuvo conmigo hasta que nació el bebé.

\subsection{Dolor (Gráfico 2)}

Algunas de las mujeres refieren que no le pusieron nada para el dolor ni antes, ni durante, ni después del parto porque no les hizo falta (solo una mujer necesitó analgesia en el parto en casa y se le administró):

CI: 6. No me tuvo que poner nada para el dolor.

\subsection{Lugar del parto (Gráfico 3)}

Algunas Cuando las mujeres referían las razones de preferir el parto en su casa era sobre todo por el hecho de ser acompañadas por sus familias. Mientras que si existían complicaciones todas veían mejor el hospital:

CI: 1. Si estás bien preparado la casa es estupendo. Claro, porque puedes estar con tu familia, estás acompañada...

Todas las mujeres que dieron a luz en casa lo hicieron en sus dormitorios y a continuación explicaron cómo se preparaban los partos allí:

CI: 3. Yo fui a la farmacia, que entonces daban una caja con todo lo que puede ser para un parto y luego lo que gastes lo pagas. Alcohol, gasas, algodón... muchas cosas... inyecciones, cosas así llevaba.

\subsection{Profesional que asiste al parto (Gráfico 4)}

Algunas de Todas las mujeres se acordaban de las matronas que eran las profesionales que más densidad de citas tienen como se observa en el gráfico 4 :

CI: 8. Benita estuvo mucho tiempo, uy era una mujer encantadora.

Tras el parto las matronas solían ir unos días a las casas a cuidar de las mujeres y los niños y ver si todo iba bien:

CI: 5. Venía dos o tres días, a ver el ombliguico. Venía a ver al crio y el primer día lo bañó ella. Y a nosotras nos lavaba, nos aseaba.

Otros profesionales asistieron partos en casa aunque en una proporción muy inferior, como es el caso del médico y el comadrón. Y en otras ocasiones, aunque muy pocas, eran los propios familiares los que se encargaban.

Con respecto al control durante el embarazo algunas de las mujeres refieren haber tenido algún control antes del parto.

\subsection{Dolor (Gráfico 5)}

Algunas de Los partos en casa fueron todos normales. Solo hubo un parto instrumentado:

\section{CI: 8. Con los fórceps me lo sacó.}

En cuanto a la episiotomía, tampoco solía hacerse en casa. Por el contrario, varias mujeres tuvieron desgarro pero no tuvieron ningún problema posteriormente ni molestias.

CI: 7. Tuve un pelín de desgarro y dijo el 
médico "si no quieres no es necesario que te ponga puntos, porque esto no tiene importancia".

En los partos en casa lo normal era que la amniorrexis fuese espontánea y tras este hecho era cuando se avisaba a la matrona para que fuera al domicilio:

CI: 5. A media noche o antes, ya a la madrugada me levanté a orinar y entonces saliendo para el aseo se me rompió.

\section{Parto en el hospital}

En el anexo $\mathrm{V}$ se recogen las gráficas correspondientes a las categorías de la familia "Parto en el hospital" según densidad de citas.

\subsection{Acompañamiento (Gráfico 6)}

En los partos en el hospital, normalmente no dejaban entrar a nadie e incluso restringían mucho las visitas en el post parto. Esto repercutía muy negativamente en el estado de ánimo de la mujer:

CI: 7. No, nadie, no había nadie, el crío nació solo, sin nadie. Y la verdad es que se pasa apuro.

\subsection{Dolor (Gráfico 7)}

En el hospital si solían dar medicación para el dolor, aunque dependía también del hospital que fuera y de los profesionales:

CI: 1. En el último, yo me acuerdo que pedía algo porque me dolía mucho y "no se puede dar nada, no se puede dar nada" y yo decía pero jaunque sea una aspirina! Y recuerdo que me dieron una aspirina infantil que eso na de na, básicamente.

Los partos más dolorosos y los que más dolores provocaban cuando ya había nacido el bebé eran los inducidos.

\subsection{Lugar del parto (Gráfico 8)}

En Las razones de que las mujeres de esta época fueran a dar a luz al hospital solían ser que ya tenían seguridad social (aunque dos mujeres que también la tenían, siguieron prefiriendo dar a luz en su casa), que habían tenido algún problema durante el embarazo (accidente, ganancia excesiva de peso, etc.), que querían que se le pusiese la epidural o por razones de seguridad:

CI: 1. Tenía acceso al seguro de enfermedad y nada, me tocó el momento del parto y fuimos al hospital.

En cuanto a la descripción del hospital, una mujer refiere muy malos recuerdos de las instalaciones, lo cual no favorecía que ésta se sintiese más cómoda, arropada y en un buen ambiente para dar a luz:

CI: 1. Que por cierto era horrible. Aquello parecía la casa de la bruja.

\subsection{Profesional que asiste al parto (Gráfico 9)}

En el hospital, las mujeres encontraron tanto con buenas como con malas matronas. Es importante incidir en el daño que hace cuando estás en una situación como es dar de parto, en la cual lo estás pasando mal, que los profesionales no te apoyen y te dejen de lado:

CI: 1. Es tan importante, yo que se una palabra cálida... y tú lo sabes, que encima que estás pasándolo mal.

Por otra parte, la buena praxis de las enfermeras del hospital transmitía una tranquilidad muy necesaria a las mujeres en esos momentos:

CI: 1. La comadrona no se agobió, para nada, no se agobió. Metió las manos, le dio el tajo y salió. Fue una obra de arte.

Son llamativas las experiencias de algunas mujeres en el hospital que refieren que estaban completamente solas a la hora del parto o posteriormente:

CI: 6. En el hospital de Marina, pues te arreglaban la cama, unas monjas o lo que fuera, se largaban y por allí no aparecía nadie.

\subsection{Técnicas usadas en el parto (Gráfico 10)}

Lo más significativo de esta categoría es que en el hospital había más episiotomías 
que en las casas, pero no de manera significativa, y que las inducciones del parto eran mucho más recurrentes, lo que provocaba que duraran mucho más tiempo y que los dolores durante éste fueran muchísimo mayores:

CI: 7. Y empezaron a darme pastillas debajo de la lengua cada media hora. Solo pastillas debajo de la lengua, nada más. Claro y entonces sí, entonces empezaron los dolores y dolores.

En el anexo VI se expone un gráfico con las principales diferencias existentes dependiendo del ámbito de realización del parto.

\section{RESULTADOS}

Centraremos la discusión en las diferencias observadas en los cuidados que se realizaban en los partos en casa y en el hospital.

Todas nuestras mujeres coinciden en que el acompañamiento es un aspecto muy importante a tener en cuenta durante el parto. Las mujeres que pudieron estar acompañadas de sus familiares en los partos en casa se sintieron mucho mejor que las que, por el contrario, estuvieron solas en la sala de partos del hospital. El calor familiar, el tener una mano que coger cuando lo estás pasando mal, el apoyo emocional que te provocan unas palabras de ánimo y, por supuesto, el tener alguien al lado cuando ya tienes a tu recién nacido para que te ayude cuando aun no puedes levantarte de la cama, son aspectos que para las mujeres adquieren una vital importancia, los cuales aportan una infinidad de beneficios $y$ permiten que las mujeres califiquen su parto como satisfactorio o no. Al igual que en nuestro estudio, diversos autores han descrito la necesidad de las mujeres de tener un acompañante que les ayude en su parto (Drew, Salmon y Webb, 1989) y han evidenciado que la presencia del acompañante aporta altos niveles de satisfacción de la mujer con su parto (Carmo, Moreira y Soares, 2004). La OMS (1996) reconoce como una práctica beneficiosa la presencia del acompañante que la mujer desee. En la "Guía de Práctica Clínica sobre la Atención al Parto Normal" del Ministerio de Sanidad y Política Social (2010) también se comprueba y recomienda el acompañamiento continuo ya que es beneficioso para la mujer (Gates, Hodnett, Hofmeyr, Sakala y Weston, 2013). Por lo tanto se debería favorecer que la mujer estuviese acompañada todo el tiempo por la persona que ella eligiese dados los resultados tan beneficiosos que produce tanto a nivel de resultados obstétricos como de una mejor experiencia del parto.

Respecto al dolor, no es uno de los aspectos a las que las mujeres le dieran mucha importancia durante el parto. Es decir, el hecho de que la mujer tuviese dolores durante el parto no impedía tener una experiencia global positiva del mismo. El estar acompañada por sus familiares, poder tener a su bebé tras el parto y recibir apoyo por parte de los profesionales, son variables a las que las mujeres les dan muchísima más importancia a la hora de considerar que su parto ha sido bueno. En nuestro estudio se puede comprobar que todos los partos, ya fueran en casa o en el hospital, eran dolorosos en mayor o menor medida. Pero cabe destacar que eran muchísimo más dolorosos si eran inducidos, y esta técnica solo se llevaba a cabo en el hospital. En la actualidad, diversos autores observan que la inducción aumenta la necesidad de analgesia, la anestesia epidural, la reanimación neonatal, aumenta la tasa de cesáreas y puede aumentar la proporción de partos instrumentales, fiebre intraparto, distocia de hombros, bajo peso al 
nacer y el ingreso en unidad de cuidados intensivos neonatal (Barbosa, Fernández, Junqueira y Mota, 2011). Los estudios actuales refieren que un correcto control del dolor si aumenta la satisfacción de la mujer con respecto al parto (Goodman, Mackey y Tavakoli, 2004) y en la "Guía de Práctica Clínica sobre la Atención al Parto Normal" del Ministerio de Sanidad y Política Social (2010) se recomienda satisfacer las expectativas de la mujer con respecto al alivio del dolor durante el parto. En un estudio realizado por Mas-Pons et al. (2010) se vio que las mujeres que pudieron disponer de los métodos de alivio de dolor que ellas prefiriesen presentaban un mayor grado de satisfacción en el parto. Esto sería lo ideal, ofrecer diversos métodos para controlar el dolor y que las mujeres pudiesen decidir.

El lugar del parto es otro factor que influía notablemente en las mujeres durante el parto. En las casas todo era mucho más familiar, confortable, acogedor y menos frío que en el hospital. Esta era la principal razón por las que las mujeres decidían dar a luz en su hogar. Por el contrario, elegían el hospital por razones de seguridad, por miedo a que algo fuera mal durante el parto. Sin embargo, se ha demostrado que en los partos de bajo riesgo la probabilidad de que ocurra algún problema es similar tanto en casa como en el hospital. Al igual que en nuestro estudio, diversos investigadores han manifestado que los partos en casa planeados y atendidos por una matrona titulada, tienen tasas de mortalidad muy bajas, comparables a las del hospital, y conllevan una reducción significativa de las intervenciones obstétricas (Janssen et al., 2009). Además de esto, en nuestro estudio aparece una mayor satisfacción general en un entorno físico agradable y relajante como es la casa y sentimientos negativos en entornos desagradables que no ayudan a relajarse. Hospitales descuidados, sucios, desatendidos, comparados con "la casa de la bruja" no pueden más que añadir dificultades a la hora del parto y volviendo a recalcar que aparte de que el sitio no ayuda a sentirse cómoda, la mujer solía encontrarse sola. Otros autores ya han indagado sobre la importancia que tiene este aspecto sobre la satisfacción de la mujer en el parto. Hodnett (2002) encontró que las mujeres preferían ser cuidadas durante el parto en lugares confortables y de aspecto familiar.

En España, las salas de dilatación de los hospitales eran la mayoría compartidas y gracias a la evidencia científica que ha demostrado la importancia de que sean individuales, se están modificando las estructuras físicas de las maternidades favoreciendo un parto más humanizado con un ambiente lo más parecido posible al de los partos en casa de nuestro estudio (Behruzi, Fraser, Goulet y Hatem, 2011). Diversos estudios han demostrado que el entorno físico en el que la mujer se encuentra durante la dilatación y el parto influye en su evolución (Behruzi, Fraser, Goulet y Hatem, 2011). Las salas de dilatación individuales permiten mantener la intimidad y el poder estar acompañada por la persona que la mujer elija (FADE, 2007).

El profesional que asiste el parto es uno de los puntos más importantes de nuestro estudio ya que hemos comprobado que es un factor muy influyente en los sentimientos de la mujer. En la casa nos hemos encontrado con matronas cariñosas, amables, atentas, de las que todas las mujeres se acordaban, incluso de su nombre. En el hospital, más de una mujer identificó un trato más distante por parte de los profesionales, no recordaban su nombre. En general todas estas experiencias reflejan que no hay nada más importante como una 
palabra cálida en los momentos en los que se está pasando mal. Como en nuestro estudio, otros autores ya evidenciaron la importancia del apoyo de los profesionales durante el parto. Carmo et al. (2004) observaron que el buen trato por parte de los profesionales sanitarios producía altos niveles de satisfacción de las mujeres en el parto. Para las mujeres el acompañamiento debe ser continuo, afectuoso y que respete los momentos de intimidad (Bravo et al., 2004). Sercekus y Okumus (2009) vieron que entre los miedos que relataban las mujeres en el parto estaban el no tener apoyo suficiente, sentirse solas y que los profesionales las tratasen de manera ruda e irrespetuosa. El apoyo por parte de los profesionales, sobretodo de enfermería, como es el caso de la matrona, es fundamental porque son los que más tiempo están al lado de las mujeres y no se debe descuidar el apoyo psicológico $\mathrm{y}$ emocional por las repercusiones que puede tener en ellas. La matrona es el profesional más apropiado para atender a las mujeres en el proceso del parto de bajo riesgo, numerosos estudios constatan esta afirmación (OMS, 1996; Ministerio de Sanidad y Política Social, 2010; Amundsen, Aune y Skaget, 2014).

Por último, con respecto a la técnica usada en el parto decir que en las casas normalmente no se realizaba la episiotomía y si las mujeres se desgarraban, posteriormente no tenían ninguna complicación. Además los partos no se inducían y la amniorrexis no se provocaba. Con todo esto, solo una mujer de las ocho entrevistadas tuvo problemas durante el parto en casa. Por el contrario, en el hospital se solía realizar la episiotomía, las matronas solían romper la bolsa de las aguas y había una tasa bastante elevada de inducciones al parto. Esto conlleva una medicalización del parto tanto para provocarlo como para paliar los dolores, ya que solían ser mucho mayores. Otros autores refuerzan la evidencia obtenida en nuestro estudio, demostrando que no es necesario el uso rutinario de la episiotomía y que la reducción en el número de las mismas aumenta el número de perinés íntegros o con lesiones leves (Carroli y Mignini, 2012).

Por último, concluir con que hoy en día se está volviendo a realizar un mayor número de partos en casa. Esto quizás sea porque las mujeres ya no sienten esa seguridad que percibían nuestras entrevistadas con respecto al parto en el hospital, y a que se está demostrando con evidencia científica que ambos partos son igual de seguros (siempre que sean de bajo riesgo y con un profesional cualificado) (Buitendijk et al., 2015).

\section{CONCLUSIONES}

Tras analizar los resultados y compararlos con los de otros autores, y teniendo en cuenta todo lo que nos ha aportado la experiencia de ocho mujeres que han dado a luz tanto en casa como en el hospital, podemos concluir que no hay evidencia científica de que el parto en casa no sea recomendable, siempre que sea un embarazo de bajo riesgo y que se esté muy bien preparado. Los aspectos que más valoran las mujeres a la hora de dar a luz serían: el acompañamiento por los familiares, el apoyo emocional de los profesionales, que el entorno sea agradable y acogedor. Estos aspectos se ven propiciados en el parto en casa. Sin embargo, refieren que el hospital les parece más seguro si surgieran complicaciones. 


\section{BIBLIOGRAFÍA}

- Amundsen, H.H., Aune, I. \& Skaget, L.C. (2014). Is a midwife's continuous presense during childbirth a matter of course? Midwive's experiences and thoughts about factors that may influence their continuous support of women during labour. Midwifery. 30(1), 89-95.

- Arantzamendi, M., Gordo, C., López-Dicastillo, O. \& Vivar C.G. (2010). La Teoría Fundamentada como Metodología de Investigación Cualitativa en Enfermería. Index de Enfermería, 19(4).

- Ariza, N.E., Lizarazo, J.P. \& Ospina, J.M. (2012). Programa madre canguro: una alternativa sencilla y costo eficaz para la protección de los recién nacidos prematuros o con bajo peso al nacer. Revista salud pública, 14(2), 32-45.

- Arnau, J. (2012). Estudio Exploratorio de la Humanización en la Atención Perinatal de la Mujer del Área I de Salud de la Región de Murcia (Tesis doctoral). Murcia: Universidad de Murcia.

- Barbosa, F.M., Fernández, E., Junqueira, S.M. \& Mota, L. (2011). Prácticas para estimular el parto normal. Index de Enfermería, 20(3).

- Behruzi, R., Fraser, W., Goulet, L. \& Hatem, M. (2011). The facilitating factors and barriers encountered in the adoption of a humanized birth care approach in a highly specialized university affiliated hospital. BMC Women's Health, 11(1), 53.

- Bravo, P. et al. (2004). Bienestar materno durante el proceso de parto: Desarrollo y aplicación de una escala de medición. Revista Chilena de Obstetricia y Ginecología, 73(1), 4-10.

- Buitendijk, S.E. et al. (2015). Perinatal mortality and morbidity ut to 28 days after birth among 743.070 low-risk planned home and hospital births: a cohort study based on three merged national perinatal databases. BJOG: An International Journal of Obstetrics \& Gynaecology, 122(5), 720-728.

- Carmo, M., Moreira E \& Soares, R.M. (2004). Aspects of women's satisfaction with childbirth care in a maternity hospital in Rio de Janeiro. Cadernos de Saúde Pública, 20, 52-62.

- Carroli, G. \& Mignini, L. (2012). Episiotomía para el parto vaginal. Cochrane Database of Systematic Reviews, 11.

- Creedy, D.K., Gamble J. \& Teakle, B. (2007). Women's expectations of maternity services: a communitybased survey. Journal of the Australian College of Midwives, 20(3), 115-20.

- De Miguel, J.R. \& Odriozola J.M. (2010). El parto de evolución lenta: conducta obstétrica en partos de bajo riesgo. Santander: Universidad de Cantabria.

- Federación de Asociaciones de Matronas de España (2007). Iniciativa Parto Normal. Documento de Consenso. Barcelona: FADE.
- Gates, S., Hodnett, E.D., Hofmeyr, G.J., Sakala, C. \& Weston, J. (2013). Continuous support for women during childbirth. Cochrane Database of Systematic Reviews, 15, 2.

- Goodman, P., Mackey, M.C. \& Tavakoli, A.S. (2004). Factors related to childbirth satisfaction. Journal of Advanced Nursing, 46(2), 212-219.

- Guía de Práctica Clínica sobre Atención al Parto Normal. (2010). Guías de Práctica Clínica en el SNS: OSTEBA.

- Hodnett, E. (2002). Pain and women's satisfaction with the experience of childbirth: A systematic review. American Journal of Obstetrics \& Gynecology, 186(5), 160-172.

- Janssen, P.A. et al. (2009). Outcomes of planned home birth with registered midwife versus planned hospital birth with midwife or physician. Canadian Medical Association Journal, 181(6-7), 377-383.

- Mas-Pons, R. et al. (2010). Satisfacción de las mujeres con la experiencia del parto: validación de la Mackey Satisfaction Childbirth Rating Scale. Gac Sanit, 26(3), 236-242.

- National Collaborating Centre for Women's and Children's Health. (2014). Intrapartum care: care of healthy women and their babies during childbirth. Clinical Guideline.

- Organización Mundial de la Salud. (1996). Cuidados en el parto normal: una guía práctica. Ginebra: OMS.

- Sercekus, P. \& Okumus, H. (2009). Fears associated with childbirth among nulliparous women in Turkey. Midwifery, 25(2), 155-62.

\section{ANEXOS \\ ANEXO I: GUÍA PARA LA ENTREVISTA}

Parto en casa:

¿Cómo fue el parto en casa? ¿Cómo lo vivió?

¿Qué cosas se preparaban y quién las preparaba?

¿En qué lugar de la casa dio a luz?

¿Quién lo asistió?

¿Cuánto tiempo estuvo de parto?

¿Le dieron algo para el dolor?

¿En qué postura tuvo al bebé?

¿Le cortaron durante el parto?

¿Quién estuvo con usted?

Parto en el hospital:

Hacer las mismas preguntas del parto en casa. Añadir:

¿Le pusieron medicación para que fuese más rápido?

\section{Finalmente se pregunta:}

¿Dónde se sintió mejor, en casa o en el hospital? ¿Con qué experiencia se queda? 


\section{Cultura de los Cuidados}

\section{ANEXO II: FOTOGRAFÍAS DE LAS CASAS DONDE SUCEDIERON LOS PARTOS}

En la imagen número 1 se muestra la casa donde dio a luz la entrevistada número 1 , localizada en San Javier.

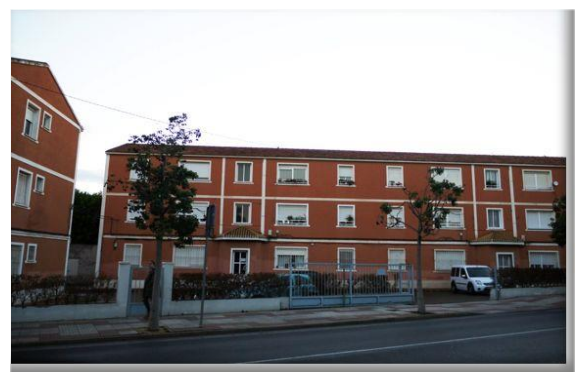

IMAGEN número 1

En la imagen número 2 se muestra la casa donde dio a luz la entrevistada número 3, localizada en Vistabella (Murcia).

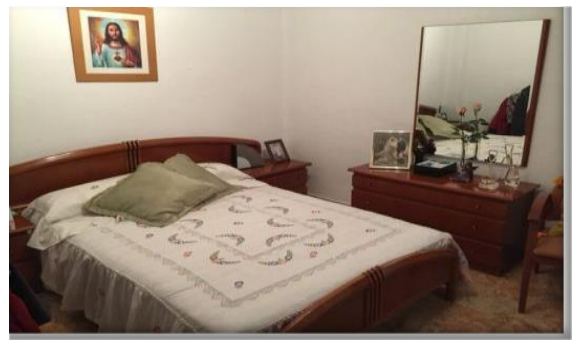

IMAGEN número 2
En la imagen número 3 se muestra la casa donde dio a luz la entrevistada número 5, localizada en Pedro del Pinatar.

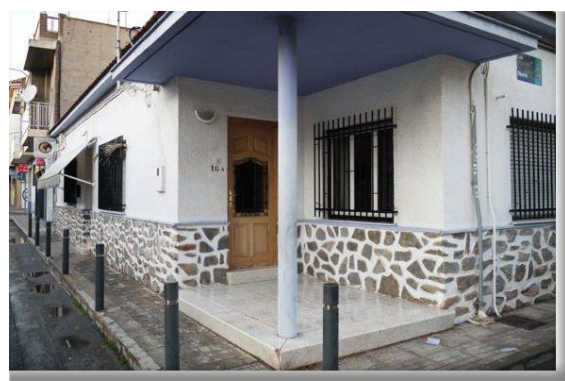

IMAGEN número 3

En la imagen número 4 se muestra la casa donde dio a luz la entrevistada número 7, localizada en Navares (Caravaca de la Cruz).

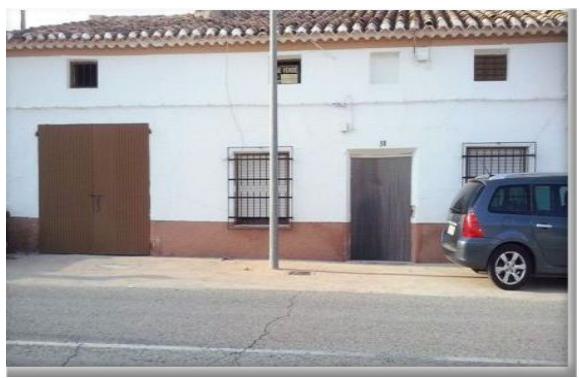

IMAGEN número 4

\section{ANEXO IV: GRÁFICOS DE LAS CATEGORÍAS DE LA FAMILIA "PARTO EN CASA"}

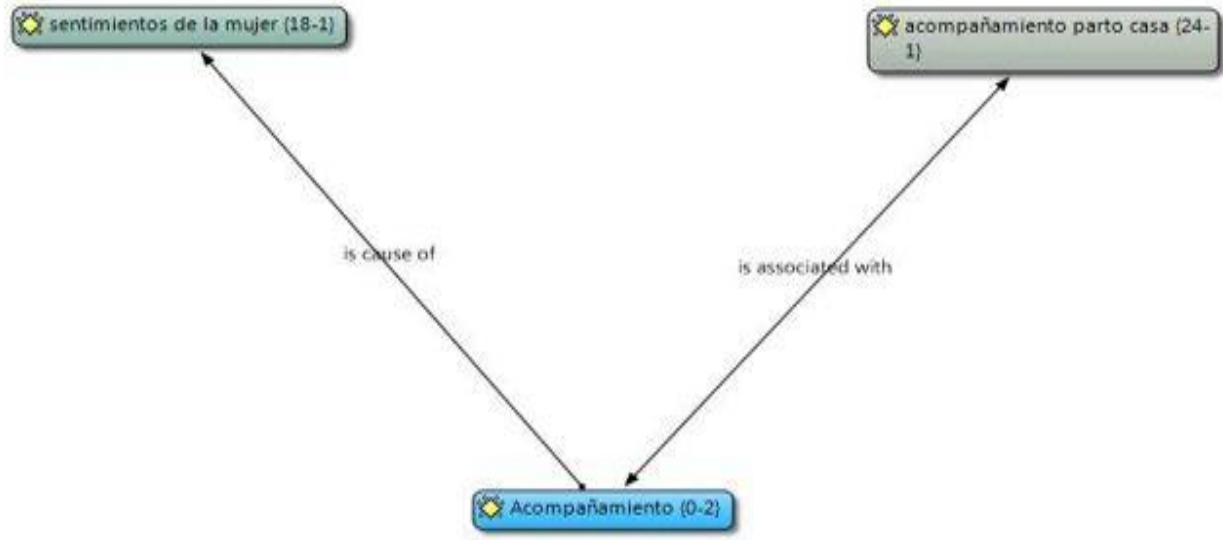

GRÁFICO 1. Acompañamiento en casa 


\section{Cultura de los Cuidados}

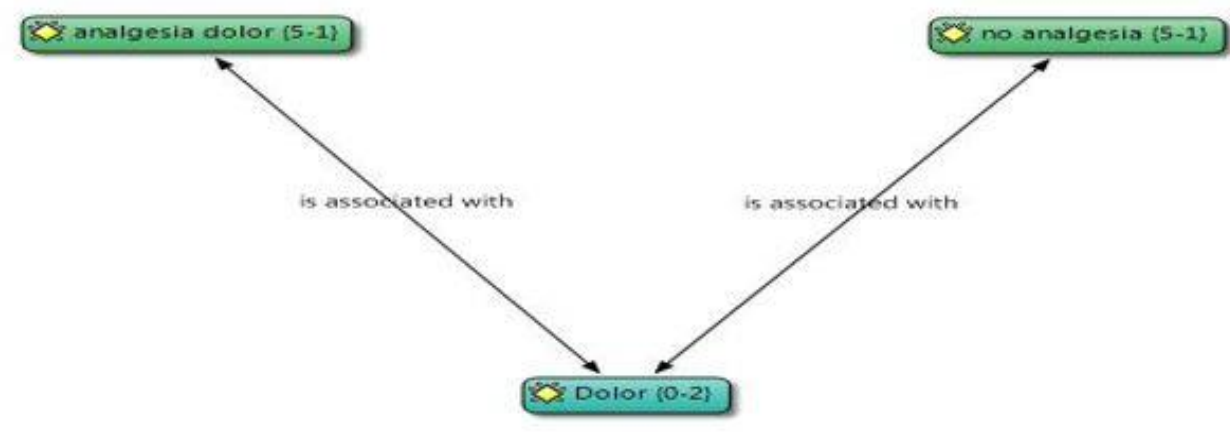

\section{GRÁFICO 2. Dolor en casa}

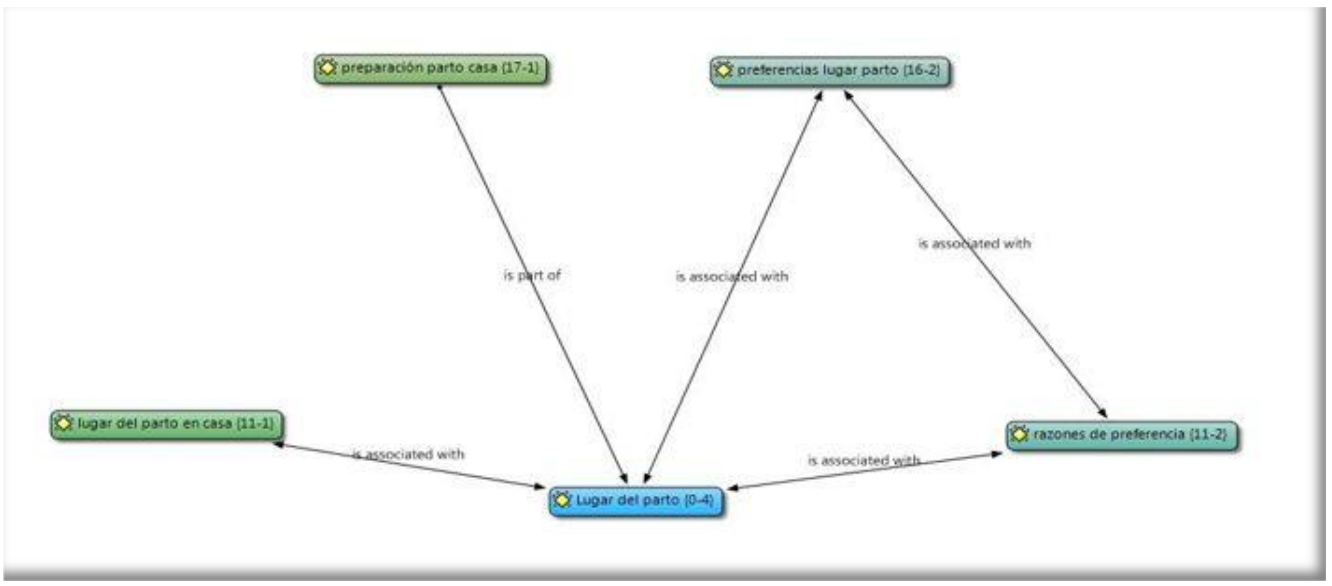

\section{GRÁFICO 3}

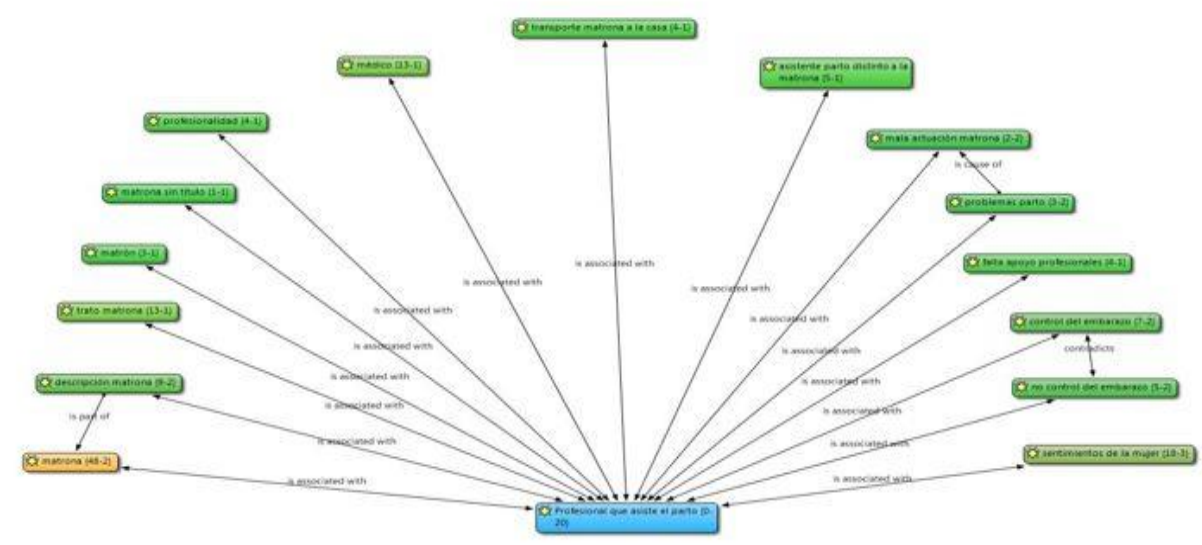

\section{GRÁFICO 4}




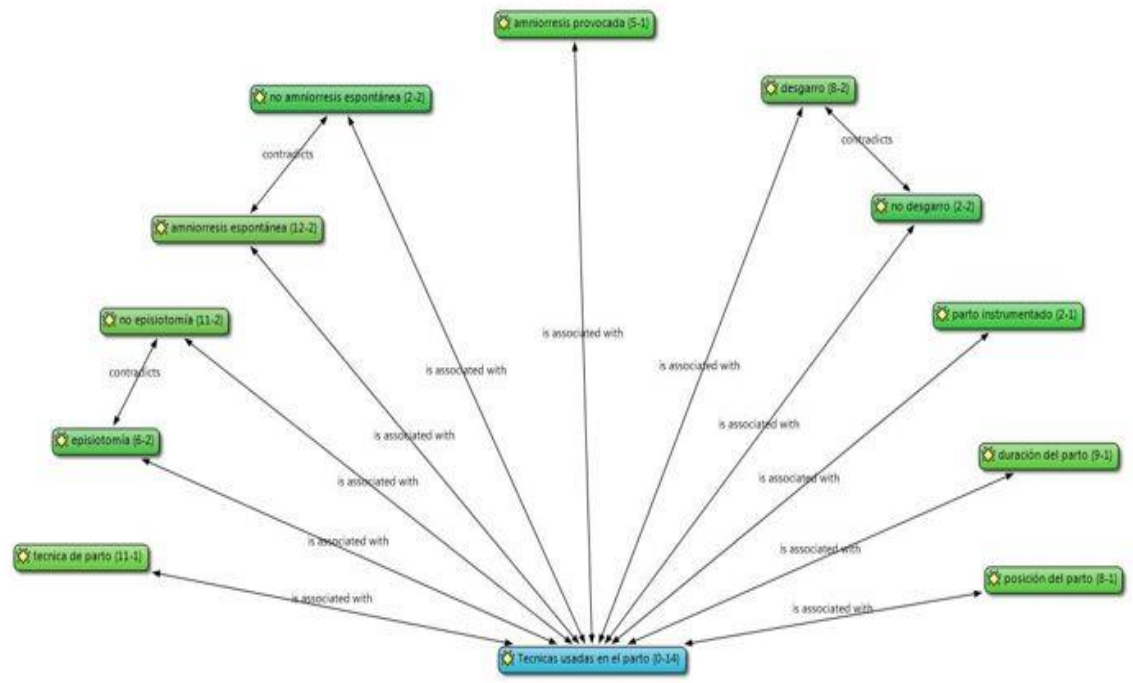

GRÁFICO 5

ANEXO V: GRÁFICOS DE LAS CATEGORÍAS DE LA FAMILIA "PARTO EN EL HOSPITAL"

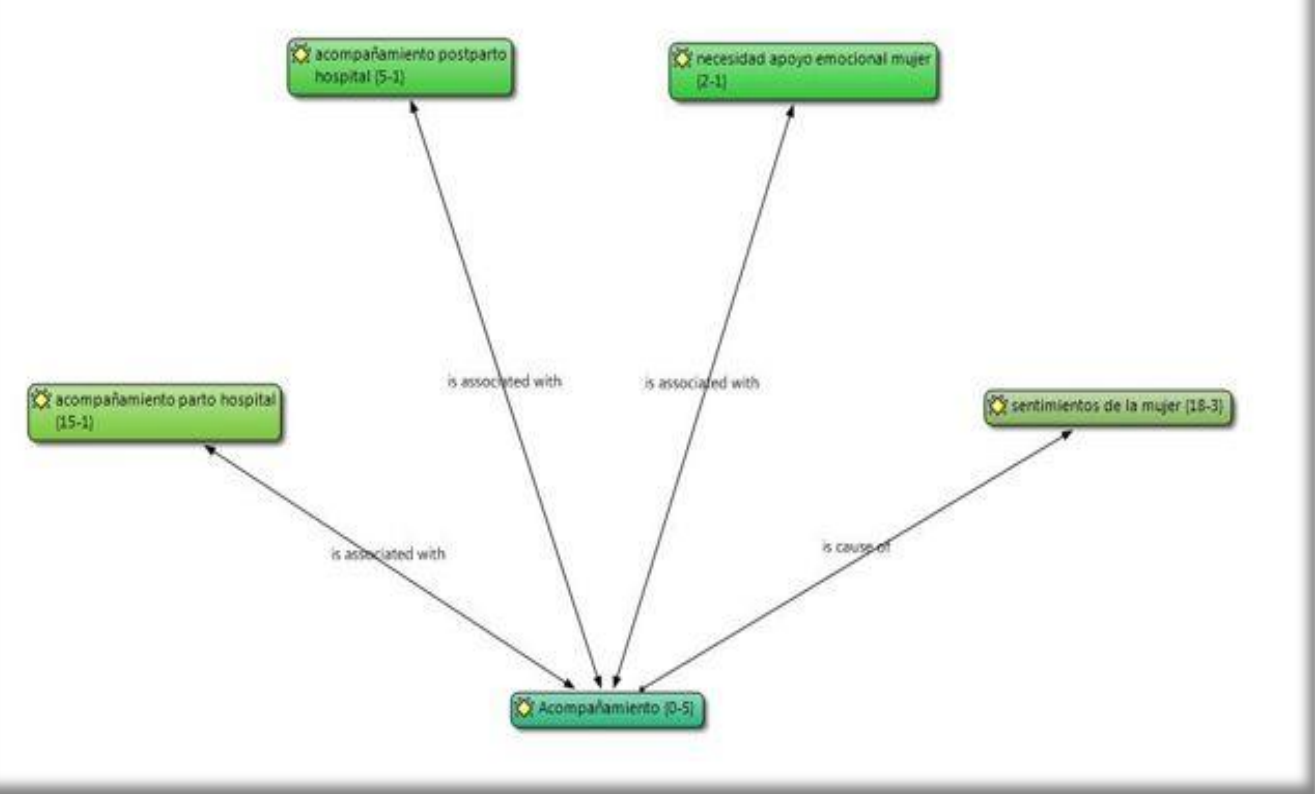

GRÁFICO 6. Acompañamiento en el hospital 


\section{Cultura de los Cuidados}

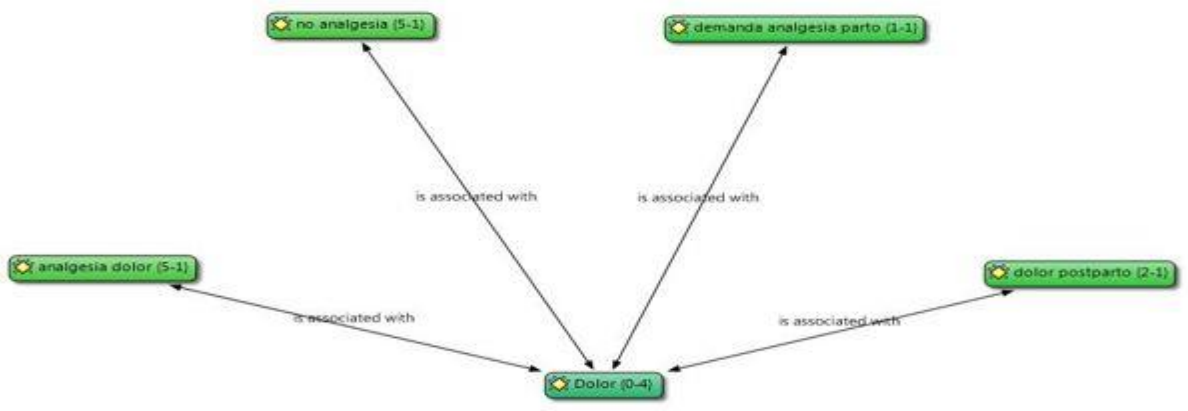

\section{GRÁFICO 7. Dolor en el hospital}

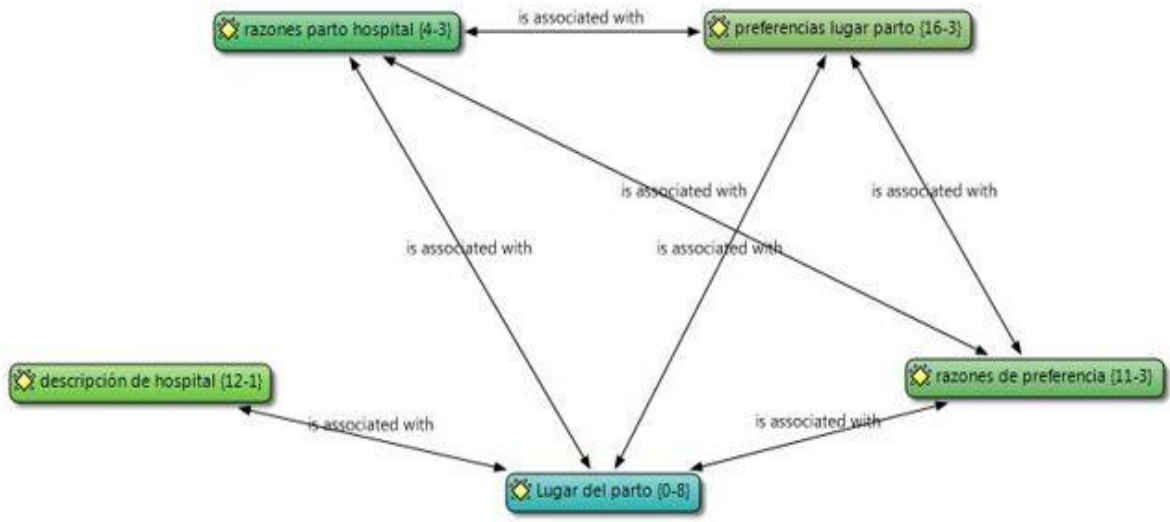

\section{GRÁFICO 8. Lugar del parto en el hospital}

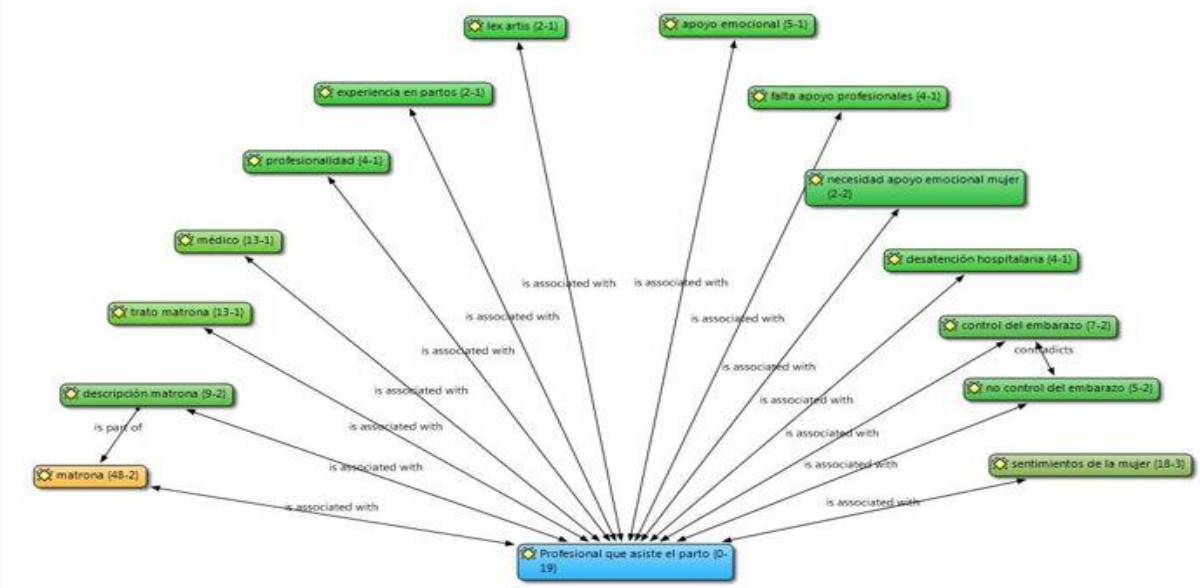

GRÁFICO 9. Profesional que asiste al parto en el hospital 


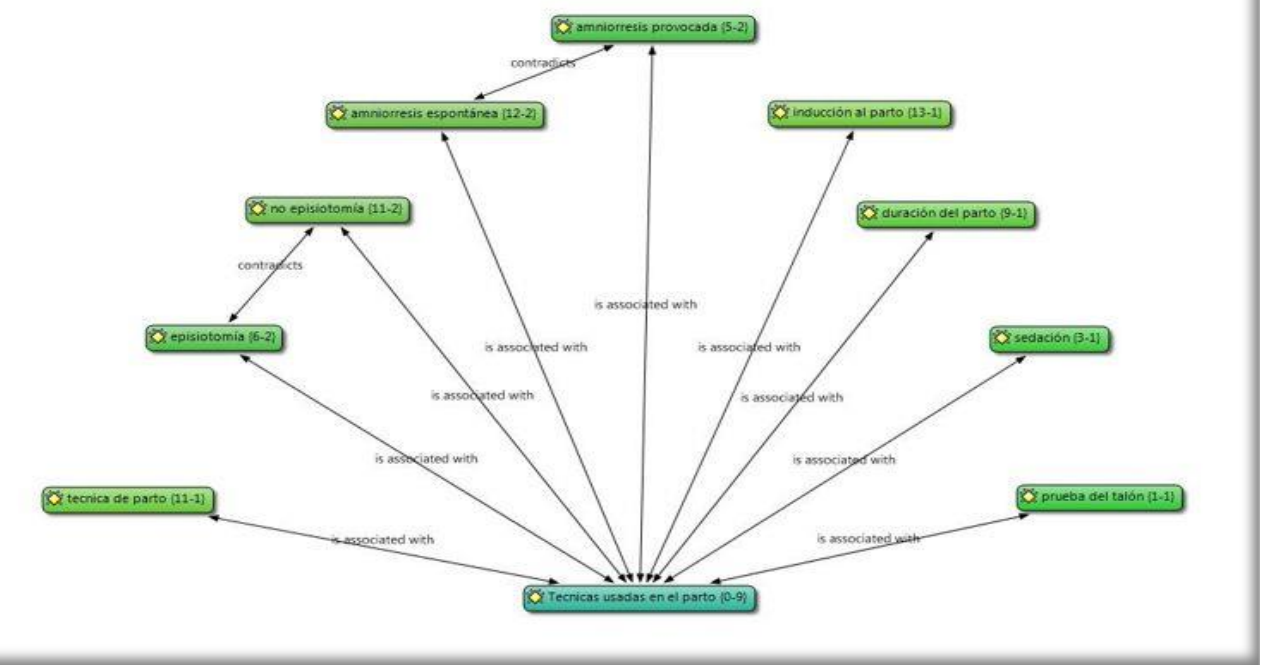

GRÁFICO 10. Técnicas usadas en el parto en el hospital

\section{ANEXO VI: DIFERENCIAS DEPENDIENDO DEL ÁMBITO DE REALIZACIÓN DEL PARTO}

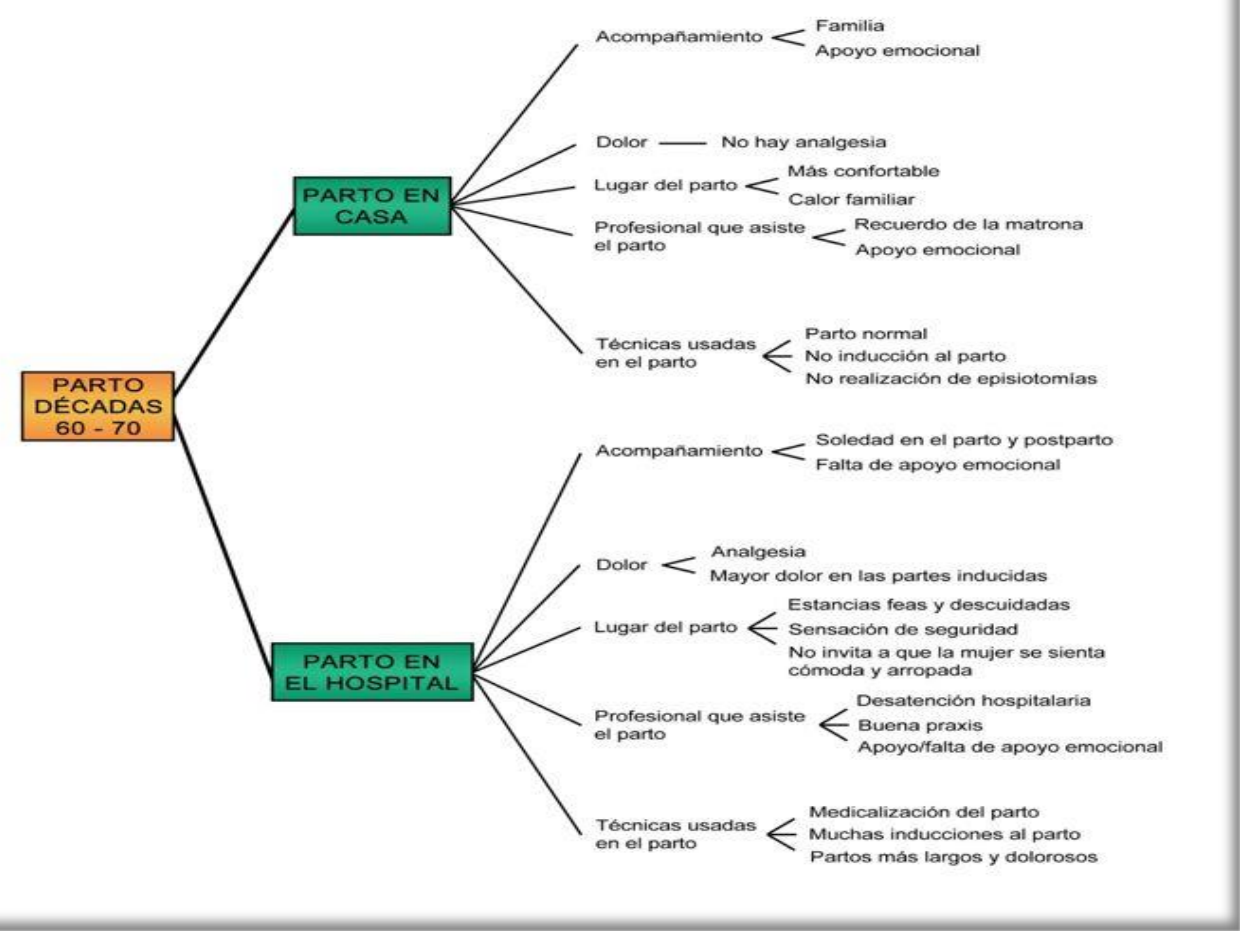

